\title{
Altered BDNF Methylation in Patients with Chronic Musculoskeletal Pain and High Biopsychosocial Complexity
}

\author{
Ariane Paoloni-Giacobino ${ }^{1} *$ \\ François Luthi $\mathbb{D I D}^{2-4, *}$ \\ Ludwig Stenz $\mathbb{D D}^{1}$ \\ Joane Le Carré2,5 \\ Philippe Vuistiner (iD ${ }^{2,5}$ \\ Bertrand Léger (iD ${ }^{2,5}$ \\ 'Medicine Faculty, Department of \\ Genetic Medicine and Development, \\ Geneva University, Geneva, Switzerland; \\ ${ }^{2}$ Institute for Research in Rehabilitation, \\ Clinique romande de réadaptation, Sion, \\ Switzerland; ${ }^{3}$ Department of \\ Musculoskeletal Rehabilitation, Clinique \\ romande de réadaptation, Sion, \\ Switzerland; ${ }^{4}$ Department of Physical \\ Medicine and Rehabilitation, Orthopaedic \\ Hospital, Lausanne University Hospital, \\ Lausanne, Switzerland; ${ }^{5}$ Department of \\ Medical Research, Clinique romande de \\ réadaptation, Sion, Switzerland
}

*These authors contributed equally to this work
This article was published in the following Dove Press journal: Journal of Pain Research

Purpose: The INTERMED instrument, which was developed to measure patient's biopsychosocial (BPS) complexity, represents a powerful diagnostic and therapeutic tool. Epigenetic changes are the interface between signals from the environment and genetic modifications, affecting gene expression, in particular, by DNA methylation of CpG dinucleotides in promotor regions of the corresponding genes. The brain-derived neurotrophic factor (BDNF) gene plays a crucial role in the central sensitization (CS) of pain. In this study, we hypothesized that chronic pain modifies the methylation levels of the BDNF gene in a manner that is interconnected with the BPS status.

Patients and Methods: Fifty-eight chronic musculoskeletal pain patients (CMSP) were enrolled in the study. DNA was extracted from blood samples, the methylation levels of 13 CpG sites in the BDNF promoter were measured by pyrosequencing, and association studies with various patient parameters and the INTERMED scores were performed.

Results: Interestingly, a negative correlation $(-0.40)$ was found between the total INTERMED scores and the average CpG methylation values of the BDNF gene, but no correlation was observed with the severity of pain, degree of anxiety, depression, or kinesiophobia and catastrophism. Moreover, the association was independent of age, sex and level of comorbidities.

Conclusion: This result shows that CMSP, in association with its biopsychosocial context, epigenetically decreases the degree of methylation of the BDNF promoter and should therefore increase the level of BDNF transcription. It also suggests a role of the INTERMED tool to detect a relationship between the BPS complexity and the epigenetic control of a target gene. The possible upregulation of BDNF expression might be, at least in part, the signal for chronic pain-induced central sensitization (CS). This could partly explain why patients with a higher level of complexity feel more pain than those with lower complexity.

Keywords: chronic pain, biopsychosocial complexity, BPS, INTERMED, brain-derived neurotrophic factor, BDNF, DNA methylation

\section{Introduction}

Biopsychosocial (BPS) complexity represents a major component of the development and maintenance of several chronic health conditions. First formulated by Engel, ${ }^{1}$ this concept, which was initially based on general system theory, has now been incorporated into modern complexity theory approaches to improve the scientific understanding of complex phenomena. ${ }^{2}$ The concept of BPS complexity has naturally been associated with health, as the human body per se is exposed to
Correspondence: Bertrand Léger Email bertrand.leger@crr-suva.ch 
multiple interactions and is composed of self-regulating physiological systems. The aim of this model is to represent human beings where biological, psychological and social factors contribute equally to the maintenance of a healthy condition or to the development of a disease. When well understood and correctly implemented, the BPS approach may represent a very powerful diagnostic and therapeutic tool for various pathologies. For instance, psychosocial factors have been shown to be better predictors of pain chronicization in low back pain patients than structural or biomechanical factors alone. ${ }^{3}$ Over the last decade, the BPS approach has been developed in conjunction with genetic and epigenetic technologies, supporting the notion that biological, psychological and social factors are indivisible.

The INTERMED tool was developed to measure a patient's BPS complexity and to screen people with complex health problems and those at risk of high use of healthcare services. It is based on the International Classification of Functioning model of illness (ICF, World Health Organization), and it integrates the dimensions of the BPS model and the relationship between patients and healthcare systems. It is considered as one of the strongest validated tools in this field. ${ }^{4}$ It has been broadly used in somatic and psychiatric contexts and in several studies on chronic pain as well. ${ }^{5-8}$ Interestingly, BPS complexity measured with the INTERMED tool has already been found to be related to biological issues. For instance, in patients with diabetes mellitus, the INTERMED scores were significantly related to the circulating HbA1c level and explained $21 \%$ of its variance among patients. ${ }^{9}$ In patients with multiple sclerosis, the INTERMED scores were also moderately related $(\mathrm{r}=0.59)$ to the neurologist rating of neurological examination abnormalities as measured with the Expanded Disability Status Scale (EDSS). ${ }^{10}$ Patients with a high degree of BPS complexity also have consistently higher pain levels than those with a low degree of complexity, regardless of the severity of the injury or disease causing the pain. ${ }^{5-8,11}$

Epigenetic changes are the interface between signals from the environment and genetic modifications affecting gene expression. Epigenetic factors modify DNA expression in different ways. They can change the level of compaction of the chromatin by histone acetylation, histone methylation and therefore regulate transcription. ${ }^{12}$ They can also directly affect DNA structure by the (de) methylation process. The latter mechanism, probably the best characterized to date, consists of the covalent addition of a methyl group to the carbon-5 of a cytosine within a $\mathrm{CpG}$ dinucleotide. Methylation in the promotor region induces a decrease in the corresponding gene expression. ${ }^{13}$ MicroRNAs are also known to eventually play a role as epigenetic modulators of gene expression. ${ }^{14}$

Chronic pain has been recently studied by combining the BPS and epigenetic approaches. In the model of chronic widespread pain, social experience and contextual factors have been shown to directly modulate gene expression. $^{15}$ In rodents, chronic inflammatory pain decreased liver $X$ receptor- $\beta$ levels in the brain, possibly by an epigenetic mechanism since it also decreased histone deacetylase 5. ${ }^{14}$ Additionally, in rodents, ethanol withdrawal increased pain sensitivity, possibly by an epigenetic mechanism because this effect was attenuated by a histone deacetylase inhibitor. ${ }^{16}$ In humans, the effect of chronic pain on DNA methylation was addressed for the first time in a study by Bruehl et al, ${ }^{17}$ which showed differentially methylated $\mathrm{CpGs}$ in immune and inflammatory-related genes in Complex Regional Pain Syndrome.

In this study, we hypothesized that chronic pain modifies methylation levels of the brain-derived neurotrophic factor $(B D N F)$ gene in a manner that is interconnected with the BPS status. The chronic musculoskeletal pain (CMSP) model was chosen.

When nociceptors are exposed to prolonged stimulation, peripheral sensitization results in a lowering of the activation threshold of the nociceptive fibres and hyperexcitability. This is one of the origins of chronic pain. Nociceptor inputs can also trigger an increase in the excitability of central nociceptive pathways, called Central Sensitization (CS). ${ }^{18}$ These mechanisms induce pain hypersensitivity, ${ }^{18}$ allodynia and the perpetuation of symptoms. ${ }^{19}$ The target brain gene chosen for this study was $B D N F$. It plays an important role in neuroplasticity and is therefore crucial for neural maintenance and repair. $^{20}$ Its signaling is part of the hypothalamic pituitary adrenal axis. ${ }^{21}$ BDNF also plays a crucial role in the CS of pain at every level. ${ }^{22-24}$

In humans, associations between methylation of $B D N F$ gene and psychiatric disorders remain controversial. A recent study failed to demonstrate such an association in the blood of patients with borderline disorders. ${ }^{25}$ However, an increased bunch of evidences tend to demonstrate an increased methylation of the $B D N F$ gene promoter in patients with depressive disorders, ${ }^{26,27}$ suicidal behaviors or those who had completed suicidal behavior, $^{28,29}$ eating disorders, childhood abuse history, ${ }^{30}$ 
and defective maternal care. ${ }^{31,32}$ Thus, BDNF seems to be associated with stressful experiences in early life and may explain later adulthood psychopathology.

In this study, we measured $B D N F$ promoter methylations levels in DNA from the blood. Indeed, as previously described, methylation analysis in peripheral blood cells is a good proxy of methylation levels in the target tissue. ${ }^{33}$ We included 58 CMSP patients and performed association studies between various patient parameters including the INTERMED scores and $B D N F$ methylation levels.

\section{Patients and Methods Study Population}

Between March 2014 and October 2017, patients with chronic musculoskeletal pain (lasting for more than 3 months) resulting from an orthopedic trauma (fractures, sprains, ...) were recruited from the Musculoskeletal Rehabilitation Department of the Clinique romande de réadaptation (Sion, Switzerland). Our sample size is comparable to that of previous studies on epigenetic modifications and pain. ${ }^{17,34}$ The exclusion criteria were as follows: a history of diabetes, toxicomania, or blood disease (ie, thalassemia) or inability to provide informed consent. The healthy control subjects, who reported no pain, were recruited among the staff of our clinic. The study protocol was approved by the Commission cantonale valaisanne d'éthique médicale (CCVEM 034/12) and was conducted according to the recommendations of the Declaration of Helsinki. Informed consent was collected from each volunteer involved in this study.

\section{Study Design}

Shortly after admission, patients were examined by a senior clinician to assess whether they met the inclusion criteria. Once they gave their informed consent, patients were scheduled for blood sampling. We used self-reported questionnaires to assess the psychological status and pain level, and clinical information was collected from the patient's medical records.

\section{Questionnaires and Clinician Rated-Tools}

The Brief Pain Inventory (BPI) is a self-administered questionnaire that allows patients to rate the severity of their pain and the degree to which their pain interferes with the common components of feeling and function. ${ }^{35} \mathrm{In}$ this study, we only focused on the items related to pain severity.
The Hospital Anxiety and Depression Scale (HADS) was used to detect states of anxiety and depression. This self-administered questionnaire was specifically developed in a hospital medical outpatient clinical setting. ${ }^{36}$

We used the Tampa Scale for Kinesiophobia (TSK) to assess pain-related fears (fears of movement and reinjury). Higher scores indicate more pain-related fears and were consistently related to higher pain and disability levels. ${ }^{37}$

We used the Pain Catastrophizing Scale (PCS) ${ }^{38}$ to investigate catastrophic thinking (rumination, magnification and helplessness), which is a risk factor for pain chronicity.

The Cumulative Illness Rating Scale (CIRS) is a clinician-oriented instrument used to assess comorbidities and illness severity. ${ }^{39}$

The DN4 questionnaire (Douleur Neuropathique en 4 Questions) was first developed to screen neuropathic pain. It is a clinician-administered questionnaire. Seven items are related to pain symptoms, and three items are associated with the neuropathic pain examination. Health professionals assess whether the sensation to touch or a pinprick (hypoesthesia) is reduced and whether light brushing of the area increases or causes pain (allodynia). This questionnaire has been broadly used and validated. ${ }^{40}$

The INTERMED instrument is a clinician-rated tool (semi-structured interview) that assesses the patient's biopsychosocial complexity. ${ }^{11,41}$ It contains 20 items grouped into 4 domains (biological, psychological, social, and healthcare system), with each one assessed over time (past, present, and prognosis). Each question is rated on a 4-point scale from 0 to 3 . A total INTERMED score ranging from 0 to 60 is calculated, whereby a higher score indicates a higher biopsychosocial complexity. It is one of the most strongly validated tools used to assess the BPS complexity. ${ }^{42}$

\section{Blood Collection}

Peripheral blood samples were collected in the morning (after an overnight fast) from the antecubital vein in $7.5 \mathrm{~mL}$ EDTA tubes (Sarstedt S-Monovette) using a butterfly device with a $21 \mathrm{G}$ needle. Immediately after collection, the tubes were frozen and kept at $-80^{\circ} \mathrm{C}$ until further processing.

\section{Methylation Analysis Procedure}

DNA was extracted from the blood using the Gentra Puregene Blood Kit (Qiagen) and diluted in $300 \mu \mathrm{L}$ of hydration solution. 
Bisulfite conversion of unmethylated cytosines to uracil was performed on $2 \mu \mathrm{g}$ of DNA using the EZ DNA Methylation Kit (Zymo Research, Irvine, CA, USA). Bisulfite-treated samples were resuspended in M-elution buffer and were stored at $-20^{\circ} \mathrm{C}$. Methylation levels of $13 \mathrm{CpG}$ sites in BDNF promoter I were measured by pyrosequencing. The PCR amplification of a $349 \mathrm{bp}$ amplicon encompassing $13 \mathrm{CpG}$ sites was performed with as described in Stenz et al, $2015^{33}$ (described as "Assay B"). PCR reactions were performed in $25 \mu \mathrm{L}$ final volume containing $50 \mathrm{ng}$ bisulfite-treated DNA, $1 \times$ PCR buffer, $1.6 \mathrm{mM} \mathrm{MgCl}_{2}, 200 \mu \mathrm{M}$ dNTP, $0.2 \mu \mathrm{M}$ forward primer, $0.2 \mu \mathrm{M}$ Reverse primer, and 2 units of Hot Start Taq DNA polymerase (HotStart Taq DNA Polymerase Kit, Qiagen). PCR amplification conditions are described in Stenz et al, 2015. ${ }^{33}$ A templatefree negative control was added to each PCR plate and agarose gel electrophoresis, to check for nonspecific amplification, was performed. Negative controls were also pyrosequenced as well as different dilutions $(0 \%$, $25 \%, 50 \%, 75 \%, 100 \%)$ from fully unmethylated $(0 \%)$ and fully methylated (100\%) genomic DNA standards (EpiTect PCR Control DNA, Qiagen). PCR products were immobilized onto streptavidin-coated sepharose beads (Fisher Scientific) using a Vacuum preparation tool (Biotage), washed in $70 \%$ ethanol, and denatured in $0.2 \mathrm{M} \mathrm{NaOH}$. Single-stranded DNA was then washed in $10 \mathrm{mM}$ Tris-acetate $\mathrm{pH} 7.6$ before being dispensed into $20 \mathrm{mM}$ Tris, $2 \mathrm{mM} \mathrm{MgAc} c_{2}$ containing $15 \mathrm{pmol}$ of forward primer in $40 \mu \mathrm{L}$ final volume. The resulting single-stranded DNA molecules were sequenced by a PSQ 96 MA (Qiagen) using the corresponding substrates, enzymes and nucleotides (PyroMark Gold Q96 SQA Reagents, Qiagen).

\section{Statistical Analysis}

The differences between the groups were evaluated by the Wilcoxon-Mann-Whitney rank-sum test. We then assessed the associations between the methylation values of $\mathrm{CpG}$ sites and the different questionnaire or evaluation scores using Spearman correlation coefficients. Since these associations might be influenced by other factors, such as age, gender or comorbidities, we next used linear regression models to adjust the associations between $\mathrm{CpG}$ values and questionnaire scores for these potential confounding variables. To improve the normality of the distributions and the linearity of the associations, the $\mathrm{CpG}$ methylation values were transformed using a negative reciprocal square root function. All analyses were performed with Stata 16.0 (Stata Corp, College Station, TX, USA).

\section{Results}

Eighteen controls and 58 patients agreed to participate in the study. No difference was observed between patients and controls for age and sex. However, CMSP patients displayed a higher BMI $(\mathrm{P}<0.01)$. Our patients were mainly middleaged men who suffered from chronic pain subsequent to a musculoskeletal injury. Their sociodemographic and psychological characteristics are listed in Table 1. In $18 \%$ of the cases, a neuropathic component, assessed by the DN4 questionnaire, was present. On average, patients reported a moderate level of pain severity and did not display any catastrophism, anxiety or depressive symptoms. ${ }^{43}$ We observed a low to moderate level of kinesiophobia in our population (Table 1).

In a preliminary experiment, our controls and our CMSP patients' average $\mathrm{CpG}$ methylation values of the $B D N F$ gene promoter were compared. Due to the relatively small sample size, we did not compare each site individually. The methylation values were significantly $(\mathrm{p}<0.005)$ 1.9-fold increased

Table I Clinical Characteristics of the Participants

\begin{tabular}{|c|c|c|}
\hline Characteristics & $\begin{array}{l}\text { Patients } \\
(\mathrm{N}=58)\end{array}$ & $\begin{array}{l}\text { Controls } \\
(N=18)\end{array}$ \\
\hline Age (years) & $41(13)$ & 40 (II) \\
\hline Sex F (percent) & $12(2 \mid \%)$ & 7 (39\%) \\
\hline BMI & $27(5)$ & $24(3)^{* *}$ \\
\hline $\begin{array}{l}\text { Time since injury (median in } \\
\text { days) }\end{array}$ & $436(39-533 I)$ & - \\
\hline DN4 $\geq 4$ & II (I8\%) & - \\
\hline Pain location & & - \\
\hline - Upper limb & $18(31 \%)$ & \\
\hline - Lower limb & 27 (47\%) & \\
\hline - Back & $11(19 \%)$ & \\
\hline - Polytrauma & $2(3 \%)$ & \\
\hline INTERMED Score $(0-60)$ & $20.6(6.2)$ & - \\
\hline BPI $(0-10)$ & $4.6(2.0)$ & - \\
\hline HADS anxiety $(0-2 \mathrm{I})$ & $8.6(4.5)$ & - \\
\hline HADS depression $(0-2 I)$ & $6.7(4.0)$ & - \\
\hline TSK (I7-68) & $42.8(8.2)$ & - \\
\hline PCS (0-52) & $19.4(11.4)$ & - \\
\hline CIRS (0-56) & $4.1(2.5)$ & - \\
\hline
\end{tabular}

Notes: The values are expressed as mean (standard deviation), median (range) or number $(\%) ; *{ }^{*} \mathrm{p}<0.01$.

Abbreviations: BMI, body mass index; DN4, Douleur Neuropathique en 4 Questions; BPI, Brief Pain Inventory; HADS, Hospital Anxiety and Depression Scale; TSK, Tampa Scale of Kinesiophobia; PCS, Pain Catastrophizing Scale; CIRS, Cumulative IIIness Rating Scale. 


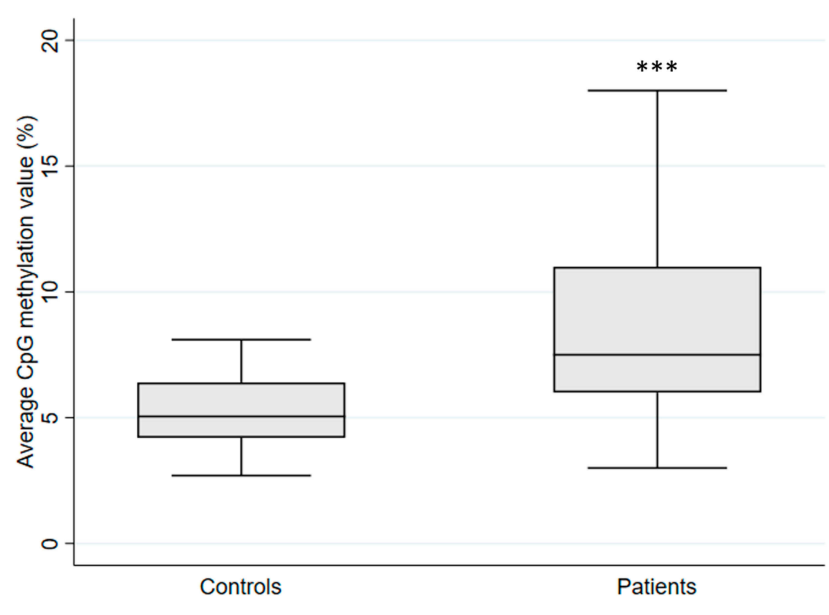

Figure I Average CPG methylation values of controls and CMSP patients. $* * *{ }^{2}<0.005, \mathrm{n}=18$ controls and 58 patients.

in the CMSP patients as compared with the controls (Figure 1). Then, a cross-sectional study was performed within the CMSP patients, targeting various pain variables. Interestingly, a negative moderate correlation was found between the total INTERMED score $(-0.40)$ and the average CpG methylation values of $B D N F$ (Table 2).

A high level of BPS complexity measured via the INTERMED score was associated with lower average CpG methylation values of BDNF (Table 3). This association was independent of potential confounding variables such as age, sex and comorbidities. There was no difference in the methylation level of $B D N F$ reported to the localization of the trauma $(\mathrm{p}=0.16)$.

\section{Discussion}

The epigenetic machinery causes modulation of gene expression to occur without any change in the DNA

Table 2 Correlations Between the Average CPG Methylation Values and the Independent Variables

\begin{tabular}{|l|l|l|}
\hline & $\begin{array}{l}\text { Spearman } \\
\text { Correlation } \\
\text { Coefficient }\end{array}$ & $\begin{array}{l}\mathbf{9 5 \%} \\
\text { Confidence } \\
\text { Interval }\end{array}$ \\
\hline INTERMED score & $-0.40 * *$ & -0.65 to -0.10 \\
Time since injury & -0.12 & -0.39 to 0.16 \\
BPI & -0.11 & -0.40 to 0.17 \\
TSK & 0.11 & -0.13 to 0.35 \\
HADS anxiety & -0.06 & -0.33 to 0.21 \\
HADS depression & -0.04 & -0.30 to 0.22 \\
PCS & -0.02 & -0.29 to 0.21 \\
\hline
\end{tabular}

Notes: ${ }^{* *} \mathrm{p}<0.01, \mathrm{n}=58$.

Abbreviations: BPI, Brief Pain Inventory; TSK, Tampa Scale of Kinesiophobia; HADS, Hospital Anxiety and Depression Scale; PCS, Pain Catastrophizing Scale.
Table 3 Average CpG Methylation Values of the BDNF Multivariate Analysis Model

\begin{tabular}{|l|l|l|l|}
\hline Outcome & Variable & $\begin{array}{l}\text { Regression Coefficient } \\
(\mathbf{9 5} \% \mathbf{C l})\end{array}$ & p-value \\
\hline $\begin{array}{l}\text { BDNF } \\
\text { CpG }\end{array}$ & INTERMED & $-0.004(-0.008$ to -0.001$)$ & $0.02 *$ \\
& score & $0.000(-0.001$ to 0.003$)$ & 0.43 \\
& Age & $0.041(-0.001$ to 0.097$)$ & 0.14 \\
& Sex & $-0.008(-0.018$ to 0.002$)$ & 0.13 \\
\hline & CIRS & \multicolumn{2}{|l}{} \\
\hline
\end{tabular}

Notes: *p<0.05, $n=58$.

Abbreviations: BDNF, brain-derived neurotrophic factor; CIRS, Cumulative IIlness Rating Scale; $\mathrm{Cl}$, confidence interval.

nucleotide sequence in response to environmental exposure. Nutritional factors, toxins and even human interactions have been shown to induce epigenetic changes. An important feature of epigenetic changes is that, despite the mechanisms of abolishment and reprogramming, they can be transmitted through generations.

Chronic pain, due to its strong impact on the quality of life, should produce epigenetic effects. However, this interesting hypothesis was the object of only rare studies, which showed that chronic pain can modify the methylation pattern of families of genes. ${ }^{15,17}$

The present study took advantage of the validated INTERMED tool, which allows evaluation of patient BPS complexity with the best possible accuracy. This tool takes into account parameters that may impact the epigenome, such as the psychosocial environment. The INTERMED method is based on a semi-structured interview that is only designed for patients and has never been used or validate to evaluate healthy volunteers.

The epigenetic effects of CMSP were focused on the $B D N F$ gene promoter, which has been shown to be differentially methylated upon exposure to psychosocial perturbations. $^{21}$

Our study can be divided into two parts. First, we performed a comparison with a control group to study a possible global effect of pain on $B D N F$ promoter methylation. It showed that a panel of patients with pains of various natures displayed on average an increased $B D N F$ promoter methylation as compared with controls (Figure 1). It should be kept in mind that our 2 groups are heterogeneous and that it cannot be excluded that other variables than pain contributed to the observed effect.

Second, the cross-sectional analysis performed inside the pain group represents the main part of the study. It showed (Table 2) that the BPI score, i.e. the severity of pain, was not correlated with $B D N F$ promoter methylation. 
Furthermore, the degree of anxiety, depression, kinesiophobia, pain catastrophizing and the time since injury also were not associated with $B D N F$ promoter methylation. However, the INTERMED score was moderately correlated with low levels of $B D N F$ promoter methylation. This result shows that BPS complexity epigenetically decrease the degree of $B D N F$ promoter methylation and should therefore increase the level of transcription of this gene.

A linear regression statistical approach (Table 3) confirmed the negative association of the INTERMED score with $B D N F$ promoter methylation and allowed the elimination of potential confounders such as age, sex or levels of comorbidities.

The fact that CMSP, taking into account its BPS complexity, is associated with a decrease in $B D N F$ promoter methylation suggests that it has an epigenetic impact opposite of that of other psychosocial stressors. ${ }^{21,31}$ A similar result was obtained by Sosanya et $\mathrm{al}^{23}$ who reported an increase in BDNF expression in the rat prefrontal cortex due to stress-induced post-injury pain.

The upregulation of BDNF is associated with higher levels of BPS complexity implied by the results of this study might induce a deleterious pain hypersensitization. ${ }^{22-24}$ BDNF capacity to initiate CS pain is considered maladaptive in chronic pain. ${ }^{22}$ Benefits for clinical practice and therapeutic perspectives of this study can be speculated: these results support a useful role for the INTERMED tool in the screening of pain patients at risk of central sensitization. Moreover, our study demonstrated a potential interaction between methylation level of BDNF and BPS complexity in CMSP patients. Over the last decades, most studies on this BPS model have focused on the psychosocial aspects, with reduced attention paid to the biological ones. The fact that BPS complexity influences methylation of BDNF gene opens a new perspective for the clinician to diagnose or cure these patients. With the rapid development of treatments, acting through modulation of epigenetic modifications, our approach may turn out to be promising. ${ }^{44}$

\section{Limitations}

Like many studies performed in the field of epigenetics, our protocol is mainly limited by its cross-sectional design that does not allow to exclude the possibility that the level of $B D N F$ methylation may evolve over time. Pain chronicization is a progressive mechanism and is associated with several other health-related disorders that might appear in a time-dependent manner (ie, anxiety, depression ...) and could influence the $B D N F$ methylation pattern. It would be of prime importance to assess, in a longitudinal study, the possible temporal changes in methylation of the BDNF gene associated with the process of pain chronicization.

The second limitation comes from the particular nature of the patients included in this study. If they are representative of the general population admitted to our rehabilitation center, they mainly report a minor to moderate level of pain. In a further study, it could be interesting to recruit patients reporting a higher level of pain in order to determine if pain intensity might be associated with methylation levels. Moreover, as our population is primarily composed of men it only includes a very low number of female patients, which may limit generalization of the results.

A methodological limitation is that hydroxymethylation of cytosine $(5 \mathrm{hmC})$ was not discriminated from methylation of cytosine in bisulfite-pyrosequencing. Evidence suggests that $5 \mathrm{hmC}$ is a secondary epigenetic mark with a complex role as a transcription regulator and an important role in the brain. ${ }^{45}$ However, the traditional bisulfite treatment sequencing method we used does not distinguish between $5 \mathrm{mC}$ and $5 \mathrm{hmC}$. Including a measurement of cytosine hydroxymethylation in a further study might be of interest.

\section{Conclusion}

To our knowledge, this study is the first to investigate $B D N F$ methylation in a human model of CMSP. Pain seemed to be associated with increased levels of $B D N F$ promoter methylation and, inside the CMSP group, the patient complexity, assessed by the INTERMED score, was associated with decreased levels of BDNF promoter methylation. This study being cross-sectional, no conclusion can be drawn on causal links between epigenetic methylation and pain hypersensitization. However, interesting hypotheses can be discussed. The downregulation of $B D N F$ expression putatively induced by chronic pain itself might be a feed-back mechanism appearing with time when pain chronicizes and that would tend to reduce pain in good prognosis patients. The upregulation of $B D N F$ expression associated with BPS complexity might be the signal for CS. Whatever the mechanism, the results of the present study suggest that a link between complexity and pain severity might be $B D N F$ expression. Therefore, a better chance of positive evolution would depend on a low level of BDNF in the brain. 


\section{Abbreviations}

BPS, biopsychosocial; CS, central sensitization; CMSP, chronic musculoskeletal pain; BDNF, brain-derived neurotrophic factor; $\mathrm{CpG}$, cytosine-phosphate-guanine; BPI, Brief Pain Inventory; HADS, Hospital Anxiety and Depression Scale; TSK, Tampa Scale for Kinesiophobia; PCS, Pain Catastrophizing Scale; CIRS, Cumulative Illness Rating Scale; DN4, Douleur Neuropathique en 4 Questions.

\section{Data Sharing Statement}

All datasets used and analyzed during the current study are available from the corresponding author on reasonable request.

\section{Ethics Approval and Informed Consent}

The study protocol was approved by the Commission cantonale valaisanne d'éthique médicale (CCVEM 034/12) and was conducted according to the recommendations of the Declaration of Helsinki. Informed written consent was obtained from each patient involved in this study.

\section{Acknowledgments}

We thank the nursing teams of the Clinique romande de réadaptation, Sion, Switzerland, who performed the blood sampling and collected the clinical information of the patients.

\section{Author Contributions}

All authors made substantial contributions to conception and design, acquisition of data, or analysis and interpretation of data; took part in drafting the article or revising it critically for important intellectual content; gave final approval of the version to be published; and agree to be accountable for all aspects of the work.

\section{Disclosure}

The authors report no conflicts of interest in this work.

\section{References}

1. Engel GL. The need for a new medical model: a challenge for biomedicine. Science. 1977;196(4286):129-136. doi:10.1126/science. 847460

2. Melchert TP. 1 - the Need for a Unified Conceptual Framework in Professional Psychology. Elsevier; 2011.

3. Pincus T, Burton AK, Vogel S, Field AP. A systematic review of psychological factors as predictors of chronicity/disability in prospective cohorts of low back pain. Spine (Phila Pa 1976). 2002;27(5): E109-120. doi:10.1097/00007632-200203010-00017

4. Marcoux V, Chouinard MC, Diadiou F, Dufour I, Hudon C. Screening tools to identify patients with complex health needs at risk of high use of health care services: a scoping review. PLoS One. 2017;12(11):e0188663. doi:10.1371/journal.pone. 0188663
5. Luthi F, Stiefel F, Gobelet C, Rivier G, Deriaz O. Rehabilitation outcomes for orthopaedic trauma individuals as measured by the INTERMED. Disabil Rehabil. 2011;33(25-26):2544-2552. doi:10.31 09/09638288.2011.579223

6. Scerri M, de Goumoens P, Fritsch C, Van Melle G, Stiefel F, So A. The INTERMED questionnaire for predicting return to work after a multidisciplinary rehabilitation program for chronic low back pain. Joint Bone Spine. 2006;73(6):736-741. doi:10.1016/j.jbspin.2005. 12.012

7. Vouilloz A, Deriaz O, Rivier G, Gobelet C, Luthi F. Biopsychosocial complexity is correlated with psychiatric comorbidity but not with perceived pain in complex regional pain syndrome type 1 (algodystrophy) of the knee. Joint Bone Spine. 2011;78(2):194-199. doi:10.1016/j.jbspin.2010.07.006

8. Stiefel FC, de Jonge P, Huyse FJ, et al. INTERMED-an assessment and classification system for case complexity. Results in patients with low back pain. Spine (Phila Pa 1976). 1999;24(4):378-384. doi:10.1097/00007632-199902150-00017

9. Fischer CJ, Stiefel FC, De Jonge P, et al. Case complexity and clinical outcome in diabetes mellitus. A prospective study using the INTERMED. Diabetes Metab. 2000;26(4):295-302.

10. Hoogervorst EL, de Jonge P, Jelles B, et al. The INTERMED: a screening instrument to identify multiple sclerosis patients in need of multidisciplinary treatment. J Neurol Neurosurg Psychiatry. 2003;74(1):20-24. doi:10.1136/jnnp.74.1.20

11. Stiefel FC, de Jonge P, Huyse FJ, et al. "INTERMED": a method to assess health service needs. II. Results on its validity and clinical use. Gen Hosp Psychiatry. 1999;21(1):49-56. doi:10.1016/S01638343(98)00061-9

12. Sabari BR, Zhang D, Allis CD, Zhao Y. Metabolic regulation of gene expression through histone acylations. Nat Rev Mol Cell Biol. 2017;18(2):90-101. doi:10.1038/nrm.2016.140

13. Bird A. DNA methylation patterns and epigenetic memory. Genes Dev. 2002;16(1):6-21. doi:10.1101/gad.947102

14. Sato F, Tsuchiya S, Meltzer SJ, Shimizu K. MicroRNAs and epigenetics. FEBS J. 2011;278(10):1598-1609. doi:10.1111/j.17424658.2011.08089.x

15. Kerr JI, Burri A. Genetic and epigenetic epidemiology of chronic widespread pain. J Pain Res. 2017;10:2021-2029. doi:10.2147/JPR. S143869

16. Pradhan AA, Tipton AF, Zhang H, Akbari A, Pandey SC. Effect of histone deacetylase inhibitor on ethanol withdrawal-induced hyperalgesia in rats. Int $J$ Neuropsychopharmacol. 2019;22(8):523-527. doi:10.1093/ijnp/pyz031

17. Bruehl S, Gamazon ER, Van de Ven T, et al. DNA methylation profiles are associated with complex regional pain syndrome following traumatic injury. Pain. 2019;160(10):2328-2337. doi:10.1097/j. pain.0000000000001624

18. Woolf CJ. Central sensitization: implications for the diagnosis and treatment of pain. Pain. 2011;152(3 Suppl):S2-15. doi:10.1016/j. pain.2010.09.030

19. den Boer C, Dries L, Terluin B, et al. Central sensitization in chronic pain and medically unexplained symptom research: a systematic review of definitions, operationalizations and measurement instruments. $J$ Psychosom Res. 2019;117:32-40. doi:10.1016/j. jpsychores.2018.12.010

20. Foltran RB, Diaz SL. BDNF isoforms: a round trip ticket between neurogenesis and serotonin? J Neurochem. 2016;138(2):204-221. doi:10.1111/jnc. 13658

21. Chiba S, Numakawa T, Ninomiya M, Richards MC, Wakabayashi C, Kunugi H. Chronic restraint stress causes anxiety- and depression-like behaviors, downregulates glucocorticoid receptor expression, and attenuates glutamate release induced by brain-derived neurotrophic factor in the prefrontal cortex. Prog Neuropsychopharmacol Biol Psychiatry. 2012;39(1):112-119. doi:10.1016/j.pnpbp.2012.05.018 
22. Nijs J, Meeus M, Versijpt J, et al. Brain-derived neurotrophic factor as a driving force behind neuroplasticity in neuropathic and central sensitization pain: a new therapeutic target? Expert Opin Ther Targets. 2015;19(4):565-576. doi:10.1517/14728222.2014.994506

23. Sosanya NM, Garza TH, Stacey W, Crimmins SL, Christy RJ, Cheppudira BP. Involvement of brain-derived neurotrophic factor (BDNF) in chronic intermittent stress-induced enhanced mechanical allodynia in a rat model of burn pain. BMC Neurosci. 2019;20(1):17. doi:10.1186/s12868-019-0500-1

24. Tao W, Chen Q, Zhou W, Wang Y, Wang L, Zhang Z. Persistent inflammation-induced up-regulation of brain-derived neurotrophic factor (BDNF) promotes synaptic delivery of alpha-amino3-hydroxy-5-methyl-4-isoxazolepropionic acid receptor GluA1 subunits in descending pain modulatory circuits. J Biol Chem. 2014;289 (32):22196-22204. doi:10.1074/jbc.M114.580381

25. Thomas M, Knoblich N, Wallisch A, et al. Increased BDNF methylation in saliva, but not blood, of patients with borderline personality disorder. Clin Epigenetics. 2018;10(1):109. doi:10.1186/s13148-0180544-6

26. Kang HJ, Kim JM, Bae KY, et al. Longitudinal associations between BDNF promoter methylation and late-life depression. Neurobiol Aging. 2015;36(4):1764 e1761-1764 e1767. doi:10.1016/j.neurobiolaging. 2014.12.035

27. Ferrer A, Labad J, Salvat-Pujol N, et al. BDNF genetic variants and methylation: effects on cognition in major depressive disorder. Transl Psychiatry. 2019;9(1):265. doi:10.1038/s41398-019-0601-8

28. Keller S, Sarchiapone M, Zarrilli F, et al. Increased BDNF promoter methylation in the Wernicke area of suicide subjects. Arch Gen Psychiatry. 2010;67(3):258-267. doi:10.1001/archgenpsychiatry.20 10.9

29. Kim J-M, Kang H-J, Kim S-Y, et al. BDNF promoter methylation associated with suicidal ideation in patients with breast cancer. Int J Psychiatry Med. 2015;49(1):75-94. doi:10.1177/00912174 15574439

30. Thaler L, Gauvin L, Joober R, et al. Methylation of BDNF in women with bulimic eating syndromes: associations with childhood abuse and borderline personality disorder. Prog Neuropsychopharmacol Biol Psychiatry. 2014;54:43-49. doi:10.1016/j.pnpbp.2014.04.010

31. Moser DA, Paoloni-Giacobino A, Stenz L, et al. BDNF methylation and maternal brain activity in a violence-related sample. PLoS One. 2015;10(12):e0143427. doi:10.1371/journal.pone.0143427

32. Unternaehrer E, Meyer AH, Burkhardt SC, et al. Childhood maternal care is associated with DNA methylation of the genes for brain-derived neurotrophic factor (BDNF) and oxytocin receptor (OXTR) in peripheral blood cells in adult men and women. Stress. 2015;18(4):451-461. doi:10.3109/10253890.2015.1038992
33. Stenz L, Zewdie S, Laforge-Escarra T, et al. BDNF promoter I methylation correlates between post-mortem human peripheral and brain tissues. Neurosci Res. 2015;91:1-7. doi:10.1016/j.neures.20 14.10.003

34. Ciampi de Andrade D, Maschietto M, Galhardoni R, et al. Epigenetics insights into chronic pain: DNA hypomethylation in fibromyalgia-a controlled pilot-study. Pain. 2017;158(8):1473-1480. doi:10.1097/j.pain.0000000000000932

35. Cleeland CS, Ryan KM. Pain assessment: global use of the brief pain inventory. Ann Acad Med Singapore. 1994;23(2):129-138.

36. Zigmond AS, Snaith RP. The hospital anxiety and depression scale. Acta Psychiatr Scand. 1983;67(6):361-370. doi:10.1111/j.16000447.1983.tb09716.x

37. Kori SH, Miller RP, Todd DD. Kinesiophobia: a new view of chronic pain behavior. Pain Manag. 1990;(3):35-43.

38. Sullivan MJL, Bishop SR, Pivik J. The pain catastrophizing scale: development and validation. Psychol Assess. 1995;7(4):524-532. doi:10.1037/1040-3590.7.4.524

39. Linn BS, Linn MW, Gurel L. Cumulative illness rating scale. J Am Geriatr Soc. 1968;16(5):622-626. doi:10.1111/j.1532-5415.1968. tb02103.x

40. Bouhassira D, Attal N, Alchaar H, et al. Comparison of pain syndromes associated with nervous or somatic lesions and development of a new neuropathic pain diagnostic questionnaire (DN4). Pain. 2005;114(1-2):29-36. doi:10.1016/j.pain.2004.12.010

41. Huyse FJ, Lyons JS, Stiefel FC, et al. "INTERMED": a method to assess health service needs. I. Development and reliability. Gen Hosp Psychiatry. 1999;21(1):39-48. doi:10.1016/S0163-8343(98)00057-7

42. Luthi F, Deriaz O, Vuistiner P, Burrus C, Hilfiker R, Karniel A. Predicting non return to work after orthopaedic trauma: the Wallis Occupational Rehabilitation RisK (WORRK) model. PLoS One. 2014;9(4):e94268. doi:10.1371/journal.pone.0094268

43. Tuscher J, Burrus C, Vuistiner P, Leger B, Rivier G, Luthi F. Predictive value of the fear-avoidance model on functional capacity evaluation. J Occup Rehabil. 2018;28(3):513-522. doi:10.1007/ s10926-017-9737-7

44. Louwies T, Ligon CO, Johnson AC, Greenwood-van Meerveld B. Targeting epigenetic mechanisms for chronic visceral pain: a valid approach for the development of novel therapeutics. Neurogastroenterol Motil. 2019;31(3):e13500. doi:10.1111/nmo.13 500

45. Rustad SR, Papale LA, Alisch RS. DNA methylation and hydroxymethylation and behavior. Curr Top Behav Neurosci. 2019;42:51-82. doi:10.1007/7854_2019_104
Journal of Pain Research

\section{Publish your work in this journal}

The Journal of Pain Research is an international, peer reviewed, open access, online journal that welcomes laboratory and clinical findings in the fields of pain research and the prevention and management of pain. Original research, reviews, symposium reports, hypothesis formation and commentaries are all considered for publication. The manuscript management system is completely online and includes a very quick and fair peer-review system, which is all easy to use. Visit http:// www.dovepress.com/testimonials.php to read real quotes from published authors. 